\title{
Diabetes Mellitus and Hypertension Increase Risk of Death in Novel Corona Virus Patients Irrespective of Age: a Prospective Observational Study of Co-morbidities and COVID-19 from India
}

\author{
Anirban Gupta ${ }^{1} \cdot$ Neelabh Nayan $^{1} \cdot$ Ranjith Nair $^{1} \cdot$ Krishna Kumar $^{2} \cdot$ Aditya Joshi $^{2} \cdot$ Shivangi Sharma $^{1} \cdot$ \\ Jasdeep Singh ${ }^{3} \cdot$ Rajan Kapoor ${ }^{1}$ (I)
}

Accepted: 4 March 2021 / Published online: 8 March 2021

(C) The Author(s), under exclusive licence to Springer Nature Switzerland AG 2021

\begin{abstract}
Elderly people and people with co-morbidities have emerged as the most vulnerable group at risk of developing complications and succumbing to novel coronavirus (COVID-19) infection. We recorded the baseline demographic profile, baseline clinical and laboratory parameters, and prevalence of various co-morbidities and their effect on the prognosis of COVID-19 cases. We conducted a prospective observational study and analyzed baseline clinical and laboratory parameters and co-morbidities and their effect on severity and mortality in 710 COVID-19 cases. Seven hundred ten patients with laboratory-confirmed COVID-19 were recruited from the $28^{\text {th }}$ of March to the $31^{\text {st }}$ of August 2020. The mean age was $48.4 \pm 16.4$ years. A total of $530(74.6 \%)$ patients were male. Overall, the mean length of hospital stay was 12.7 days. In total, 645 patients( $(90.8 \%)$ were mild to moderate cases and did not require initial ICU care. Sixty-five (9.2\%) cases required initial intensive care unit care. Fifty (7\%) admitted patients succumbed to the illness. Diabetes mellitus and hypertension increased the risk of death in COVID-19 patients irrespective of age. Increasing age and co-morbidities adversely affect the prognosis of patients of COVID-19. Diabetes mellitus and hypertension increase the risk of death in COVID-19 patients and negate the incremental effect of age on death in these patients.
\end{abstract}

Keywords Diabetes $\cdot$ Hypertension $\cdot$ Co-morbidities $\cdot$ COVID-19 $\cdot$ Prognosis $\cdot$ Mortality

\section{Introduction}

The World Health Organization on March 11, 2020, declared the novel coronavirus (COVID-19) outbreak a global pandemic [1]. As per www.worldometer.info> coronavirus, until 29 Oct 2020, a total of 44,787,418 persons got infected worldwide with $1,179,410$ deaths. India has emerged as the second most affected country worldwide with $8,040,203$ cases and 120,563 deaths. In terms of percentages, India accounted

This article is part of the Topical Collection on COVID-19

Rajan Kapoor

majrkapoor@gmail.com

1 Department of Internal Medicine, Command Hospital (Eastern Command), Kolkata 700027, India

2 Department of Anaesthesia and Critical Care, Command Hospital (Eastern Command), Kolkata, India

3 Department of Pathology, Command Hospital (Eastern Command), Kolkata, India for $17.95 \%$ of the global COVID-19 burden. The good thing is that a majority of these cases are asymptomatic or mildly symptomatic [2]. Elderly people and people with comorbidities have emerged as the most vulnerable group at risk of developing complications and succumbing to this illness [3]. They are also at increased risk of requiring intensive care unit (ICU) care and critical care resources. In a resource-scarce country like India, it is imperative that we study the effects of co-morbidities on overall prognosis of COVID-19 cases to effectively allocate healthcare resources and plan the overall management strategy of COVID-19. Very few studies have been published regarding this issue especially from India.

\section{Aim \\ To study the baseline demographic profile, baseline clinical and laboratory parameters and prevalence of various co- morbidities and their effect on severity and mortality in cases of COVID-19 treated in a tertiary care hospital in eastern India}


and to correlate the prognosis of these patients based on their co-morbidities.

\section{Materials and Method}

We conducted a prospective observational study from 28 March 2020 to 31 Aug 2020. Study subjects were all consecutive patients with positive reverse transcriptase polymerase chain reaction for COVID-19 who were admitted in a dedicated COVID-19 care facility in our hospital. An informed consent was taken from all patients prior to enrolling in the study. Ethical clearance was taken from the institutional ethical committee. Case history was taken from patients and their relatives or caregivers who brought the patients. All vital parameters like pulse, blood pressure, temperature, respiratory rate, and peripheral capillary oxygen saturation ( $\mathrm{SpO} 2)$ were recorded by admitting medical officer. A baseline general and systemic examination was done and recorded in the case sheet. At baseline, blood was drawn to test complete blood count, liver function test, renal function test, serum lactate dehydrogenase, blood sugar profile, serum electrolytes, and prothrombin time and international normalized ratio. Baseline chest X-ray and electro-cardiogram were done in all cases. Selective cases underwent contrast-enhanced computed tomography of the chest to look for pulmonary involvement. Additionally, all cases were tested for C-reactive protein, serum ferritin, and d-dimer levels. All vital parameters were monitored 6 hourly by dedicated staff and findings were recorded. All co-morbidities were recorded in the case sheet. We broadly divided them as hypertension (HTN), diabetes mellitus (DM), cancer, chronic respiratory disease (RESP), cardiac disease (CD), chronic kidney disease (CKD), neurological disease (ND), surgical cases, orthopedic cases, and gynecological cases. All patients requiring dialysis underwent routine dialysis care under a dedicated dialysis team lead by a nephrologist. Also, all cases requiring intensive care unit admission and having respiratory complications were managed by a dedicated intensivist and pulmonologist. All other subspecialities were consulted for all complications related to their speciality. The data from COVID-19 patients were recorded in an excel sheet and analyzed further.

\section{Statistical Analysis}

We used the chi-square test for comparison of categorical data and the Mann-Whitney test for comparison of non-parametric continuous data. Frequency, mean, and standard deviation values were determined. Categorical variables are reported as number $(\%)$. The odds ratio and $95 \%$ confidence interval were calculated to find the association of co-morbidities with the mortality outcome. The receiver operating characteristic curve for sensitivity and specificity was determined for clinical characteristics. Tests were considered significant at a $P$ value less than 0.05. Data were analyzed using STATA, version 13.0 (Stata Statistical Software, Release 13; StataCorp LP, College Station, TX). Data visualization was done by R version 3.6.3.

\section{Results}

\section{Baseline Clinical Characteristics}

A total of 710 patients with laboratory-confirmed COVID-19 cases were recruited from the $28^{\text {th }}$ of March to the $31^{\text {st }}$ of August 2020. The mean age was $48.4 \pm 16.4$ years. Five hundred thirty $(74.6 \%)$ patients were male. Overall, the mean length of hospital stay was 12.7 days. In total, 645 patients $(90.8 \%)$ were mild to moderate cases and did not require initial ICU care. Sixty-five $(9.2 \%)$ cases required initial ICU care. Fifty (7\%) admitted patients succumbed to the illness. Table 1 analyzes the baseline clinical characteristics of all admitted cases of COVID-19 and compares them between ICU cases, vis-a-vis, non-ICU cases. On further sub-group analysis, the presence of higher age, HTN, tachycardia, tachypnoea, low baseline $\mathrm{SpO} 2$, and presence of higher grade of fever were found to have a significantly higher risk $(P<.05)$ for ICU admission. Gender had no added significance for risk for ICU admission. Mortality was significantly more in patients requiring initial ICU care $(52.3 \%$ vis-a-vis $2.4 \%, P<$ $.001)$. The duration of hospital stay was not significantly different between ICU and non-ICU cases.

\section{Correlation of Baseline Characteristics with Mortality}

We also compared the baseline clinical characteristics between survivors vis-à-vis non-survivors using receiver operating characteristic curve (Fig. 1). The presence of HTN, tachycardia, tachypnea, and fever at baseline was found to be significantly associated with mortality. Low $\mathrm{SpO} 2$ and length of stay were not found to be significantly associated with mortality.

\section{Baseline Laboratory Parameters}

The baseline laboratory parameters were compared for significant differences between the survivors vis-a-vis nonsurvivors in Table 2. Hemoglobin, absolute lymphocyte count, and serum albumin levels were found significantly lower in non-survivors. The values of total leucocyte count, absolute neutrophil count, neutrophil to lymphocyte ratio (NLR), international normalized ratio, alanine transaminase, aspartate transaminase, and lactate dehydrogenase were found 
Table 1 Baseline clinical characteristics of patients hospitalized with COVID-19

\begin{tabular}{lllll}
\hline Variables & $\begin{array}{l}\text { ICU Patients, } n=65 \\
(\%)\end{array}$ & $\begin{array}{l}\text { Non-ICU patients, } \\
n=645(\%)\end{array}$ & $\begin{array}{l}\text { Total patients, } N= \\
710(\%)\end{array}$ & $\begin{array}{l}P \\
\text { value }\end{array}$ \\
\hline Age (years) & $58.4 \pm 13.2$ & $47.6 \pm 17.5$ & $48.4 \pm 16.4$ & $<0.001$ \\
Male & $46(70.8)$ & $484(75)$ & $530(74.6)$ & 0.88 \\
Female & $19(29.2)$ & $161(25)$ & $180(25.4)$ & 0.56 \\
BP systolic( $\mathrm{mmHg})$ & $138.1 \pm 19.5$ & $102.4 \pm 12.2$ & $118.2 \pm 20.8$ & $<0.001$ \\
BP diastolic $(\mathrm{mmHg})$ & $91.4 \pm 12.3$ & $72.9 \pm 11.7$ & $81.4 \pm 16.1$ & $<0.001$ \\
Pulse & $109.4 \pm 9.7$ & $101.8 \pm 10.4$ & $102.5 \pm 10.5$ & $<0.001$ \\
Respiratory rate & $23.5 \pm 3.4$ & $20.4 \pm 1.7$ & $20.7 \pm 2.1$ & $<0.001$ \\
Body Temperature ${ }^{\circ}$ & $100.2 \pm 9.7$ & $99.1 \pm 4.1$ & $99.2 \pm 3.9$ & 0.03 \\
$\quad$ F) & $89.7 \pm 6.7$ & $95.9 \pm 4.5$ & $95.4 \pm 5.1$ & $<0.001$ \\
SPO & $12.8 \pm 8.0$ & $12.7 \pm 8.1$ & 0.56 \\
Length of hospital & $12.2 \pm 8.5$ & $16(2.4)$ & $49(6.9)$ & $<0.001$ \\
$\quad$ stay & $34(52.3)$ & & &
\end{tabular}

$B P$, blood pressure; ${ }^{\circ} \mathrm{F},{ }^{\circ}$ Fahrenheit significantly higher in non-survivors compared to those in survivors $(P<.05)$.

\section{Baseline Co-morbidities of Patients Hospitalized with COVID-19}

Out of 710 patients, 422 (59.44\%) had no co-morbidity, 202 (28.45\%) had one co-morbidity, and $86(12.11 \%)$ had 2 or more co-morbidities. Figure 2 shows the frequency of each co-morbidity. Table 3 describes the baseline co-morbidities of patients hospitalized with COVID-19 in our hospital (ICU visa-vis non-ICU). The prevalence of major co-morbidities in descending order was HTN $(n=82,12 \%)$, DM $(n=53,7.2 \%)$, cancer $(n=50,7.1 \%)$, HD $(n=49,6.7 \%)$, CKD $(n=39,5.3 \%)$, $\mathrm{ND}(n=37,5.2 \%)$, and RESP $(n=29,4.0 \%)$. We also observed that the probability of ICU admission was significantly more in cases of RESP (OR 7.7 (2.7-20.0)), DM (OR 5.7 (2.911.0)), CKD (OR 5.1 (2.2-10.9)), ND (OR 3.5 (1.4-7.8)), and HTN (OR 3.4 (1.8-6.1)).

\section{Number of Co-morbidities and Overall Outcome}

It was also observed as illustrated by a stacked bar plot in Fig. 3 dividing patients who died or recovered that lesser comorbidities resulted in better outcomes.
Fig. 1 Baseline characteristics and mortality $(N=710)$. BPsys systolic blood pressure, BPdias diastolic blood pressure, Temp temperature, RR respiratory rate, $\mathrm{SpO} 2$ peripheral capillary oxygen saturation, $R O C$ receiver operating characteristic

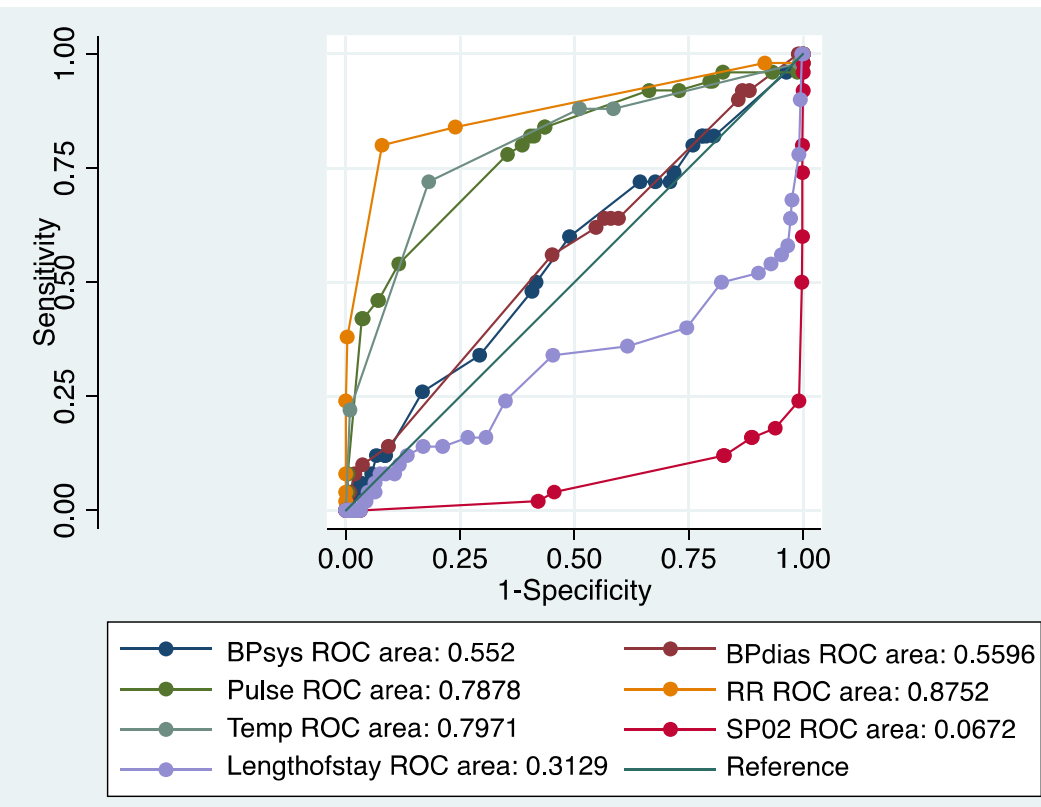


Table 2 Baseline laboratory parameters

\begin{tabular}{llll}
\hline Parameter & Survivor (mean values) & Non-survivor (mean values) & $P(\mathrm{CI})$ \\
\hline $\mathrm{Hb}(\mathrm{g} / \mathrm{dL})$ & 11.5 & 10.1 & $<0.001(1.06-1.83)$ \\
$\mathrm{TLC} \times 10^{9} / \mathrm{L}$ & 8.5 & 13.1 & $<0.001(-5.9$ to -3.4$)$ \\
$\mathrm{ANC} \times 10^{9} / \mathrm{L}$ & 6.3 & 11.2 & $<0.001(-5.9$ to -3.7$)$ \\
$\mathrm{ALC} \times 10^{9} / \mathrm{L}$ & 1.6 & 1.1 & $<0.001(0.25$ to 0.65$)$ \\
$\mathrm{NLR}$ & 6.3 & 15.5 & $<0.001(-11.5$ to -7$)$ \\
Platelet $\times 10^{9} / \mathrm{L}$ & 224 & 163 & $0.04(1$ to 120$)$ \\
$\mathrm{INR}$ & 1.15 & 1.46 & $0.003(-0.51$ to -0.10$)$ \\
$\mathrm{ALT}(\mathrm{IU} / \mathrm{L})$ & 62 & 97 & $0.011(-62$ to -8$)$ \\
$\mathrm{AST}(\mathrm{IU} / \mathrm{L})$ & 60 & 110 & $0.003(-84$ to -17$)$ \\
$\mathrm{Serum}$ albumin $(\mathrm{mg} / \mathrm{dL})$ & 3.3 & 2.7 & $<0.001(0.53$ to 0.74$)$ \\
$\mathrm{LDH}(\mathrm{IU} / \mathrm{L})$ & 690 & 1433 & $<0.001(-918$ to -568$)$ \\
\hline
\end{tabular}

$H b$, hemoglobin; $T L C$, total leucocyte count; $A N C$, absolute neutrophil count; $A L C$, absolute lymphocyte count; $N L R$, neutrophil lymphocyte ratio; $I N R$, international normalized ratio; $A L T$, alanine transaminase; $A S T$, aspartate transaminase; $L D H$, lactate dehydrogenase; $C I$, confidence interval

\section{Age and Mortality}

Age was found to be an independent predictor for the mortality as depicted in Fig. 4 with an interpretation that increase in the age of COVID-19 patients have a worse outcome.

\section{Co-morbidity and Mortality in COVID-19}

We also looked at the mortality rates of patients with various co-morbidities. Table 4 shows the statistics of co-morbidity and mortality in COVID-19. DM (OR5.9 (2.8-11.7)), CKD (OR 5.3 (2.3-11.8)), ND (OR 4.7 (1.9-10.6)), HTN (OR4.2 (2.3-7.7)), and RESP (OR $4.2(1.1-12.8)$ ) were found to have a significant risk of mortality.

\section{Effect of Age and Co-morbidities on Overall Outcome.}

We also analyzed the effect of age and co-morbidities on overall outcome as illustrated in Table 5. Higher age was significantly associated $(P<.05)$ with poorer outcome. However, the trends in diabetics and hypertensives were different. In these patients, younger age had no survival benefit. The trends in RESP were reverse. Figures 5 and 6 further illustrate the deleterious effect of DM and HTN on overall prognosis. In Fig. 5, age is plotted on the $x$-axis, while the proportion of death is plotted on the $y$-axis in this line graph. The red line depicts patients who have died and do not have DM, while the green line depicts patients who have died and have DM. Shadows around these lines depict confidence interval. It is clearly seen that in the presence of DM, younger

Table 3 Baseline co-morbidities (ICU vis-a-vis non-ICU)

\begin{tabular}{|c|c|c|c|c|}
\hline Variables & ICU patients, $n=65(\%)$ & Non-ICU patients, $n=645(\%)$ & Total patients, $N=710(\%)$ & OR $(95 \% \mathrm{CI})$ \\
\hline DM & $19(29.2)$ & $33(5.0)$ & $53(7.2)$ & $5.7(2.9-11.0)$ \\
\hline HTN & $22(33.9)$ & $65(9.9)$ & $87(12)$ & $3.4(1.8-6.1)$ \\
\hline Cancer & $3(4.6)$ & $47(7.3)$ & $50(7.1)$ & $0.62(0.12-2.1)$ \\
\hline CKD & $13(20)$ & $26(3.9)$ & $39(5.3)$ & $5.1(2.2-10.9)$ \\
\hline HD & $6(9.2)$ & $43(6.7)$ & $49(6.7)$ & $1.4(0.4-3.5)$ \\
\hline ND & $10(15.4)$ & $27(4.2)$ & $37(5.2)$ & $3.5(1.4-7.8)$ \\
\hline RESP & $9(13.9)$ & $12(1.8)$ & $21(2.9)$ & $7.7(2.7-20.0)$ \\
\hline Orthopedic cases & $1(1.6)$ & $28(4.3)$ & $29(4.0)$ & $0.36(0.008-2.2)$ \\
\hline Surgical cases & $1(1.6)$ & $35(5.2)$ & $36(4.9)$ & $0.29(0.007-1.8)$ \\
\hline Gynaecological cases & 0 & $21(3.3)$ & $21(2.9)$ & $0(0-1.8)$ \\
\hline
\end{tabular}

$D M$, diabetes mellitus; $H T N$, hypertension; $C K D$, chronic kidney disease; $H D$, heart disease; $N D$, neurological disorders; $R E S P$, chronic respiratory disease 
Fig. 2 Baseline co-morbidities. HTN hypertension, DM diabetes mellitus, HD heart disease, CKD chronic kidney disease, ND neurological disorders, RESP chronic respiratory illness

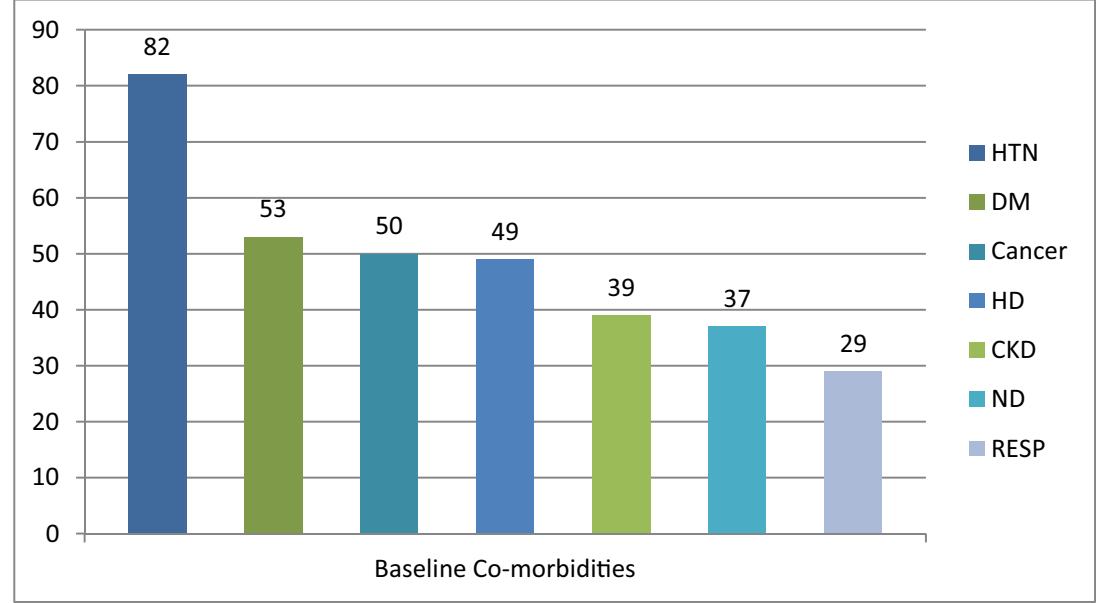

patients have an increased risk of mortality. In Fig. 6, it is seen that in the presence of HTN, the risk of mortality remains the same across the age spectrum.

\section{Discussion}

The COVID-19 pandemic is presently raging globally and we are facing the $2^{\text {nd }}$ and $3^{\text {rd }}$ wave of the pandemic stretching the global healthcare system to its limits. Though the virus does not distinguish between the rich and the poor, the health care facilities and abilities of the poorer countries are starkly different from the richer countries. Nowhere in the world is this truer than India which is facing the dual threat of a huge disease burden and a very poor health infrastructure. With this fact in mind, we went about documenting the cases of COVID19 in our large tertiary care facility. Ours is one of the largest single-center studies in India and we addressed the issue of co-morbidities of our patients of COVID-19 and

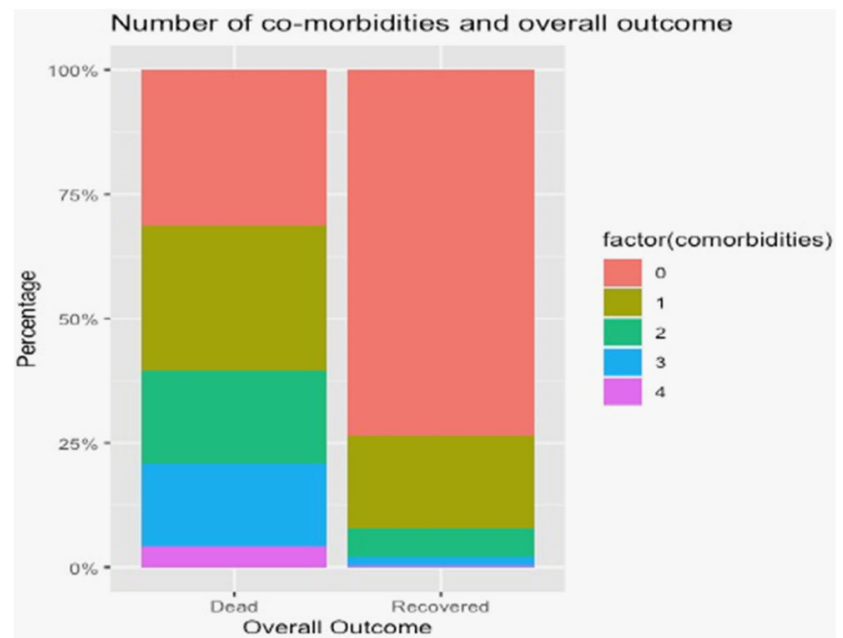

Fig. 3 Number of co-morbidities and overall outcome. Stacked bar plot showing the number of co-morbidities in two groups: dead and recovered its impact on overall prognosis of our patients. In the baseline clinical characteristics, we found that higher age, presence of tachycardia, HTN, tachypnoea with baseline lower $\mathrm{SpO}$, and higher grade of fever were associated with more chances of ICU admission. Mortality was also significantly more in cases initially admitted in ICU. Gender and duration of hospital stay were not significantly different between ICU and non-ICU cases. These findings are self-explanatory and only reiterate the fact that sicker patients at baseline required ICU management and these patients more frequently succumbed to the illness. However, lower baseline $\mathrm{SpO} 2$ did not result in higher mortality in hospitalized patients. Jiang Xie et al. from Wuhan, China [4], studied 140 patients with moderate to severe COVID-19-associated pneumonia and concluded that lower $\mathrm{SpO} 2$ correlated with higher mortality. Our cases were a mixture of presentations with COVID-19 and not only respiratory cases, and hence, our findings

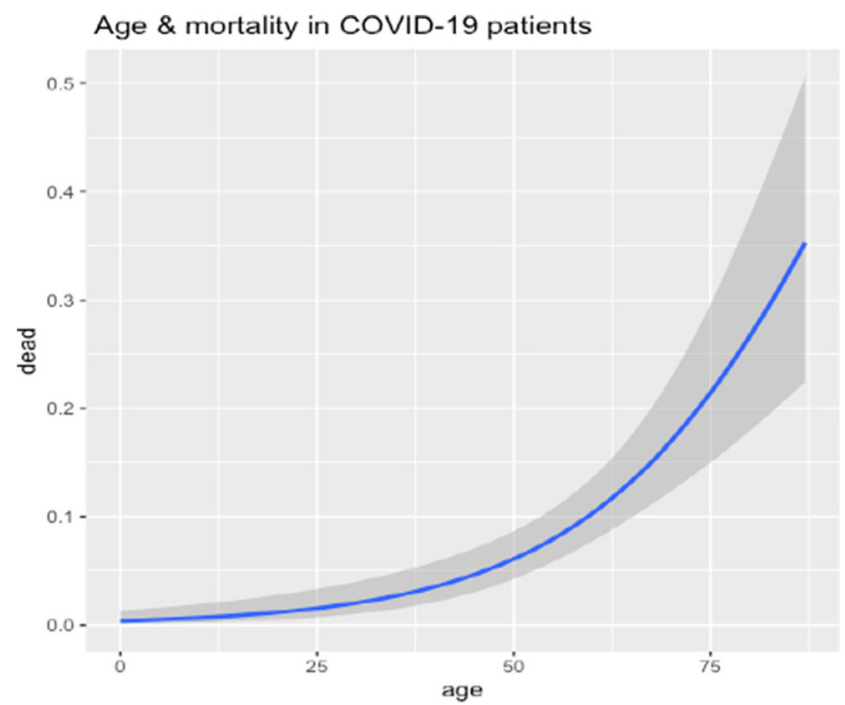

Fig. 4 Age and mortality with COVID-19 $(N=710)$. Correlation between age and mortality: On the $x$-axis is age in years and the $y$-axis depicts the proportion of death among hospitalized COVID-19 patients 
Table 4 Co-morbidity and mortality in COVID-19 $(N=710)$

\begin{tabular}{llll}
\hline Variables & Non-survivor, $n=50(\%)$ & Survivor, $N=660(\%)$ & Odds ratio $(95 \% \mathrm{CI})$ \\
\hline $\mathrm{DM}$ & $16(32.0)$ & $36(5.4)$ & $5.9(2.8-11.7)$ \\
$\mathrm{HTN}$ & $21(42.0)$ & $66(9.9)$ & $4.2(2.3-7.7)$ \\
Cancer & $5(10.0)$ & $45(6.8)$ & $1.4(0.4-3.8)$ \\
$\mathrm{CKD}$ & $11(22.0)$ & $28(4.2)$ & $5.3(2.3-11.8)$ \\
$\mathrm{HD}$ & $5(10)$ & $44(6.4)$ & $1.3(0.4-3.7)$ \\
ND & $10(20)$ & $27(4.1)$ & $4.7(1.9-10.6)$ \\
RESP & $5(10)$ & $16(2.4)$ & $4.2(1.1-12.8)$ \\
Orthopedic cases & $1(2.0)$ & $28(4.2)$ & $0.48(0.01-3.1)$ \\
Surgical cases & $2(4.0)$ & $34(5.1)$ & $0.8(0.09-3.2)$ \\
Gynaecological cases & 0 & $21(3.1)$ & $0(0-2.52)$ \\
\hline
\end{tabular}

$D M$, diabetes mellitus; $H T N$, hypertension; $C K D$, chronic kidney disease; $H D$, heart disease; $N D$, neurological disorders; RESP, chronic respiratory disease; $C I$, confidence interval of insignificant correlation of $\mathrm{SpO} 2$ with mortality are explained. Contrary to some studies indicating poorer prognosis for males $[3,5]$, our study did not reveal any difference in prognosis based on gender. We found lower hemoglobin, absolute lymphocyte count, serum albumin, higher total leukocyte count, absolute neutrophil count, NLR, international normalized ratio, alanine transaminase, and aspartate transaminase at baseline correlating significantly with higher chances of mortality. Viral infections are known to cause leucopenia with relative lymphocytosis [6] and COVID-19 infection is no different [7]. Lower hemoglobin [8] and serum albumin [9] leads to poorer outcomes in ICU patients and our findings correlate with this. Higher total leucocyte count, absolute neutrophil count, international normalized ratio, and transaminases have been previously reported in COVID-19 infection [7]. Our study correlated with poorer outcomes of patients due to the direct involvement of COVID-19 infection and its hematological effects. Higher NLR has

Table 5 Effect of age and co-morbidities on outcomes

\begin{tabular}{llll}
\hline Parameter & $\begin{array}{l}\text { Survivor } \\
\text { Mean age (years) }\end{array}$ & $\begin{array}{l}\text { Non-survivor } \\
\text { Mean age (years) }\end{array}$ & $P$ value (CI) \\
\hline Overall & 48 & 62 & $<\mathbf{0 . 0 0 1}(\mathbf{1 1 - 1 8})$ \\
DM & 62 & 62 & $0.93(-7$ to 6$)$ \\
HTN & 62 & 62 & $0.89(-5.3$ to 6.1$)$ \\
CKD & 53 & 62 & $0.08(-1$ to 18$)$ \\
ND & 53 & 63 & $0.05(0-21)$ \\
CANCER & 48 & 54 & $0.33(-9$ to 22$)$ \\
CD & 58 & 69 & $0.11(-4$ to 26$)$ \\
RESP & 67 & 57 & $0.02(-19$ to -2$)$ \\
\hline
\end{tabular}

$D M$, diabetes mellitus; $H T N$, hypertension; $C K D$, chronic kidney disease; $H D$, heart disease; $N D$, neurological disorders; RESP, chronic respiratory disease; $C I$, confidence interval been associated with poorer outcomes in COVID-19 infection [10] and our study also correlates with this. The most common co-morbidities in our patients were HTN $(n=82,12 \%), \mathrm{DM}(n=53,7.2 \%)$, cancer $(n=50,7.1 \%)$, HD $(n=49,6.7 \%), \mathrm{CKD}(n=39,5.3 \%), \mathrm{ND}(n=37,5.2 \%)$, and RESP $(n=29,4.0 \%)$. We also observed that the probability of ICU admission was significantly more in cases of RESP (OR 7.7 (2.7-20.0)), DM (OR 5.7(2.9-11.0)), CKD (OR 5.1 (2.2-10.9)), ND (OR $3.5(1.4-7.8)$ ), and HTN (OR 3.4 (1.8-6.1)). WJ Guan et al. reported HTN and $\mathrm{DM}$ as the most common co-morbidities associated with COVID-19 infection (3). They also reported that HTN,

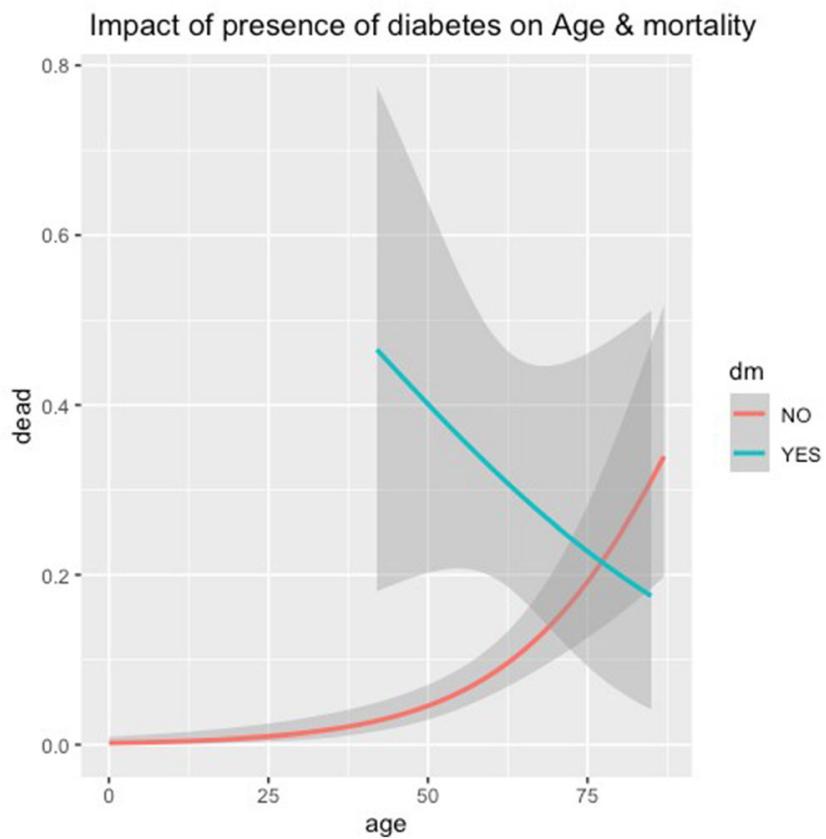

Fig. 5 Effect of diabetes on age and mortality. DM diabetes mellitus. Line graph depicting the relation between age in years ( $x$-axis) and proportion of death ( $y$-axis) among diabetics (green) and non-diabetics (red). Shaded grey areas depict confidence intervals 


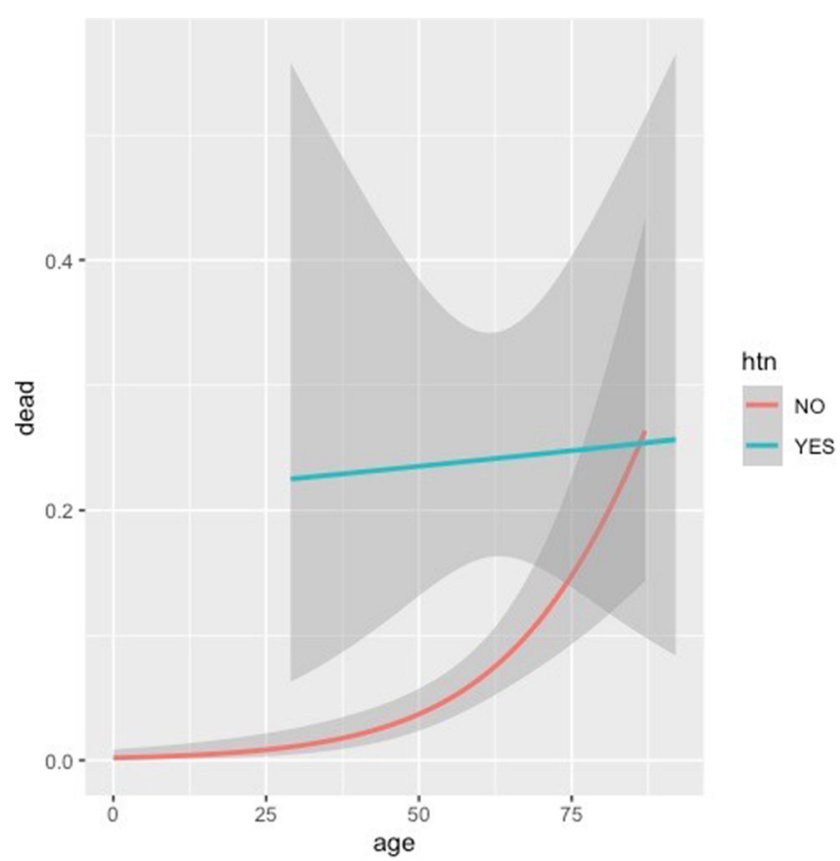

Fig. 6 Effect of hypertension on age and mortality. HTN hypertension. Line graph depicting the relation between age in years ( $x$-axis) and proportion of death ( $y$-axis) among hypertensives (green) and nonhypertensives (red). Shaded grey areas depict confidence intervals

DM, cancer, and chronic obstructive pulmonary disease were associated with poorer outcomes in COVID-19 infection (3). MS GOLD et al. in a systematic review and meta-analysis reported that HTN, DM, and respiratory diseases had poorer outcomes in COVID-19 infection [11]. In a systematic review and meta-analysis, Fang et al. reported poorer prognosis in patients with $\mathrm{CKD}$, chronic obstructive pulmonary disease, and neurological disorders [5]. Overall, our findings confirmed all these studies. Patients with two or more co-morbidities had poorer outcomes in our study as has already been reported by WJ Guan et al. [3]. Higher age as a significant risk factor for poorer outcomes has been earlier brought out in various studies $[5,12]$. Our study also report poorer outcomes in older patients with COVID-19 infection. In other words, younger age had a relatively protective effect in terms of outcome in COVID-19 infection. However, in our study, it was brought out that in patients with HTN and DM, younger age did not provide any major advantage in survival. All previous studies have reported poorer outcomes in patients of COVID-19 with DM and HTN. We analyzed the effects of age in these patients. It was always being suspected that younger patients with these co-morbidities also had a significantly higher risk of ICU admission and mortality. Cunningham et al. have reported that "in-hospital mortality rate of young patients with DM and HTN is lower than that reported for older adults with
COVID-19, but approximately double that of young adults with acute myocardial infarction. HTN and DM were common and associated with greater risks of adverse events. Young adults with more than one of these conditions faced risks comparable with those observed in middle-aged adults without them"[13] and our study conclusively proves this point.

\section{Limitations}

For the purpose of this study, the presence of comorbidities was ascertained by history and checking medical records only. However, the fact whether these comorbidities were well-controlled or poorly controlled, or recently diagnosed was not recorded at the time of admission, which can affect the final outcome in these patients.

\section{Conclusion}

To the best of our knowledge, this is one of the largest studies studying the effects of co-morbidities on the outcome of COVID-19 patients, especially from India. We have covered various aspects of COVID-19 infection and its prognosis starting from the epidemiological profile, baseline clinical and laboratory parameters, and baseline co-morbidities. It is well known that older age and multiple co-morbidities lead to poorer outcomes in COVID-19 patients. However, the major finding in our study is that the presence of DM and HTN makes younger COVID-19 patients equally susceptible to poorer outcomes. These major co-morbidities negate the incremental effect of age on death in these patients. This is important in countries like India, the "diabetes capital of the world," with a large proportion of young population. With no definitive treatment or vaccine available, public health experts in our country should target young people with comorbidities and reinforce that social distancing, face masks, and other approaches to prevent transmission are as important in young adults as in older persons.

Abbreviations COVID-19, Novel coronavirus; ICU, Intensive care unit; $\mathrm{SpO}$ 2, Peripheral capillary oxygen saturation; HTN, Hypertension; DM, Diabetes mellitus; RESP, Chronic respiratory disease; CD, Cardiac disease; CKD, Chronic kidney disease; ND, Neurological disease; NLR, Neutrophil to lymphocyte ratio

Author Contribution AG designed the study and wrote the manuscript. $\mathrm{NN}$ collected data. $\mathrm{RN}$ collected data and edited the manuscript. KK, AJ, and SS collected data. JS performed required tests and also collected data. RK collated all data into an excel sheet and analyzed data and edited the manuscript.

Data Availability Available 
Code Availability Not applicable

\section{Declarations}

Ethics Approval Institutional ethical committee approval was taken.

Consent to Participate Informed consent was taken from all participants.

Consent for Publication Taken from the institute.

Conflict of Interest The authors declare no competing interests.

\section{References}

1. Cucinotta D, Vanelli M. WHO declares COVID-19 a pandemic. Acta Biomed. 2020;91(1):157-60.

2. Day M. Covid-19: four fifths of cases are asymptomatic, China figures indicate. BMJ. 2020;369(April):m1375. Available from:. https://doi.org/10.1136/bmj.m1375.

3. Guan WJ, Liang WH, Zhao Y, Liang HR, Chen ZS, Li YM, et al. Comorbidity and its impact on 1,590 patients with Covid-19 in China: a nationwide analysis. Eur Respir J. 2020;55(5).

4. Xie J, Covassin N, Fan Z, Singh P, Gao W, Li G, et al. Association Between Hypoxemia and Mortality in Patients With COVID-19. Mayo Clin Proc. 2020;95(6):1138-47. Available from:. https://doi. org/10.1016/j.mayocp.2020.04.006.

5. Fang X, Li S, Yu H, Wang P, Zhang Y, Chen Z, et al. Epidemiological, comorbidity factors with severity and prognosis of COVID-19: a systematic review and meta-analysis. Aging (Albany NY). 2020;12(13):12493-503.

6. Herbinger KH, Hanus I, Beissner M, Berens-Riha N, Kroidl I, Von Sonnenburg F, et al. Lymphocytosis and lymphopenia induced by imported infectious diseases: a controlled cross-sectional study of 17,229 diseased German travelers returning from the tropics and subtropics. Am J Trop Med Hyg. 2016;94(6):1385-91.

7. Letícia de OT S, Sousa NL, das Graças CM, Romana AD, de Barros PM. COVID-19: Review and hematologic impact. Clin Chim Acta. 2020;510:170-176.

8. Rawal G, Kumar R, Yadav S, Singh A. Anemia in intensive care: a review of current concepts. J Crit Care Med. 2016;2(3):109-14.

9. Kim YS, Sol IS, Kim MJ, Kim SY, Kim JD, Kim YH, et al. Serum albumin as a biomarker of poor prognosis in the pediatric patients in intensive care unit. Korean J Crit Care Med. 2017;32(4):347-55.

10. Yang AP, Liu JP, Tao WQ, Li HM. The diagnostic and predictive role of NLR, d-NLR and PLR in COVID-19 patients. Int Immunopharmacol. 2020;84:106504.

11. Gold MS, Sehayek D, Gabrielli S, Zhang X, McCusker C, BenShoshan M. COVID-19 and comorbidities: a systematic review and meta-analysis. Postgrad Med. 2020;00(00):1-7. Available from:. https://doi.org/10.1080/00325481.2020.1786964.

12. Grasselli G, Zangrillo A, Zanella A, Antonelli M, Cabrini L, Castelli A, et al. Baseline characteristics and outcomes of 1591 patients infected with SARS-CoV-2 admitted to ICUs of the Lombardy Region, Italy. JAMA. 2020;323(16):1574-81.

13. Cunningham JW, Vaduganathan M, Claggett BL, Jering KS, Bhatt AS, Rosenthal N, et al. Clinical outcomes in young US adults hospitalized with COVID-19. JAMA Intern Med. 2020:17-9.

Publisher's Note Springer Nature remains neutral with regard to jurisdictional claims in published maps and institutional affiliations. 